\title{
Self-Assembly and Nanostructures in Organogels Based on a Bolaform Cholesteryl Imide Compound with Conjugated Aromatic Spacer
}

Ti-Feng Jiao ${ }^{1,2}{ }^{*}$, Feng-Qing Gao ${ }^{1}$, Xi-Hai Shen ${ }^{1,3}$, Qing-Rui Zhang ${ }^{1}{ }^{*}$, Xian-Fu Zhang ${ }^{3}$, Jing-Xin Zhou ${ }^{1}$ and Fa-Ming Gao ${ }^{1}$

1 Hebei Key Laboratory of Applied Chemistry, School of Environmental and Chemical Engineering, Yanshan University, Qinhuangdao 066004, China; E-Mails: gaofengqing619@126.com (F.-Q.G.); shenxihai2007@126.com (X.-H.S.); zhoujingxin@ysu.edu.cn (J.-X.Z.); fmgao@ysu.edu.cn (F.-M.G.)

2 State Key Laboratory of Metastable Materials Science and Technology, Yanshan University, Qinhuangdao 066004, China

3 College of Physics and Chemistry, Hebei Normal University of Science and Technology, Qinhuangdao 066004, China; E-Mail: zhangxf111@hotmail.com

* Authors to whom correspondence should be addressed; E-Mails: tfjiao@ysu.edu.cn (T.-F.J.); zhangqr@ysu.edu.cn (Q.-R.Z.); Tel.: +86-335-8387-744 (Q.-R.Z.);

Fax: +86-335-8061-569 (Q.-R.Z.).

Received: 7 November 2013; in revised form: 1 December 2013 / Accepted: 9 December 2013 / Published: 12 December 2013

\begin{abstract}
The self-assembly of small functional molecules into supramolecular structures is a powerful approach toward the development of new nanoscale materials and devices. As a class of self-assembled materials, low weight molecular organic gelators, organized in special nanoarchitectures through specific non-covalent interactions, has become one of the hot topics in soft matter research due to their scientific values and many potential applications. Here, a bolaform cholesteryl imide compound with conjugated aromatic spacer was designed and synthesized. The gelation behaviors in 23 solvents were investigated as efficient low-molecular-mass organic gelator. The experimental results indicated that the morphologies and assembly modes of as-formed organogels can be regulated by changing the kinds of organic solvents. Scanning electron microscopy and atomic force microscopy observations revealed that the gelator molecule self-assemble into different aggregates, from wrinkle and belt to fiber with the change of solvents. Spectral studies indicated that there existed different $\mathrm{H}$-bond formations between imide groups and
\end{abstract}


assembly modes. Finally, some rational assembly modes in organogels were proposed and discussed. The present work may give some insight to the design and character of new organogelators and soft materials with special structures.

Keywords: nanostructure; self-assembly; organogel; bolaform amphiphile; cholesteryl compound; spacer

\section{Introduction}

In recent years, Organogels have attracted a great deal of interest due to wide applications on materials, drug delivery, agents, sensors, water purification, etc. [1-21]. In nature, organogels are various three-dimensional aggregates with micrometer-scale lengths and nanometer-scale diameters immobilizing the flow of liquids. The main driving forces responsible for the formation of supramolecular organogels are specific or non-covalent interactions, such as the dipole-dipole interaction, van der Waals forces, hydrogen bonding, $\pi-\pi$ stacking, and host-guest interaction [22-27]. Interestingly, complementary hydrogen bonding patterns play a very important role in forming various nanoarchitectures, and their application in the fabrication of organogels has been investigated [28-30]. In addition, although gels are originally found in polymer systems, there has recently been an increasing interest in low-molecular mass gelators (LMMGs) [31-33]. Such organogels have some advantages over polymer gels: the molecular structure of the gelator is defined, and the gel process is usually reversible. Such properties make it possible to design various functional gel systems and produce more complicated and controllable nanostructures [34-37].

As an important class of LMMGs, cholesteryl-based imide compounds have been extensively studied for more than two decades, and even today they still remain as an actively investigated class of compounds [38-40]. The mentioned "imide structure" is referred to " $\mathrm{O}=\mathrm{C}-\mathrm{NH}$ ", which have demonstrated an important role in forming strong hydrogen bonding and regulating organized aggregates. These organogels have unique directional self-association through van der Waals interactions in the aggregates of the gelators. For example, Shinkai et al. prepared a number of dicholesterol derivatives bearing various functional linkers as versatile gelators [41-45] and obtained inorganic materials possessing unique structures by using the corresponding gels as templates. Recently, Fang's group reported some gel systems, with functionalized cholesteryl derivatives as gelators, and especially, some of the gelators' gel oil from oil-water mixtures [46-49]. In addition, in our reported works, the gelation properties of some cholesterol imide derivatives consisting of different substituent groups have been investigated [50-52]. We found that a subtle change in the headgroups, spacers, and molecular shapes in molecular skeletons could produce a dramatic change in the gelation behaviors of all compounds.

In this paper, as a continuous work, we have designed and synthesized this bolaform cholesteryl imide compound with large conjugated aromatic spacer. In this compound, the phenanthridine segment with additional phenyl group in spacer was symmetrically attached to cholesterol substituent headgroups to show bolaform molecular skeleton. According to previous reports, 3,8-diamino-6-phenylphenanthridine, as phenyl-phenanthridine dye with a large $\pi$-conjugated 
structure and strong luminescence, were used as materials to synthesize some nanoparticles and show special aggregates [53]. So in present work, this unit was added to the spacer in bolaform amphiphile in order to perform some new characters in organogels in line with those of amphiphiles with hydrophobic or hydrophilic spacers [51]. We have found that the resulting compound was an efficient gelator and could form different organogels in organic solvents. Characterization of these organogels by scanning electron microscopy (SEM) and atomic force microscopy (AFM) revealed different nanostructures of the aggregates in the gels. We have investigated the assembly modes in the microstructures of such organogels and found different kinds of hydrogen bond interactions between imide groups and assembly units.

\section{Results and Discussion}

\subsection{Gelation Behaviors}

Firstly, the gelation performances of the imide compound abbreviated as $\mathrm{CH}-\mathrm{PY}$ in 23 solvents are listed in Table 1. Examination of the table reveals that the compound is an efficient gelator. CH-PY can gel in 10 kinds of solvents, such as n-butanol, n-pentanol, isopentanol, isooctanol, cyclopentanone, cyclohexanone, nitrobenzene, n-butyl acrylate, dimethylformamide (THF), and tetrahydrofuran (DMF). The corresponding photographs of organogels of $\mathrm{CH}-\mathrm{PY}$ in different solvents are shown in Figure 1. The present data shown in Table 1 indicate that the aromatic spacer in molecular skeletons have a profound effect upon the gelation abilities of the present studied imide compound, which is similar to some systems in our previous report about organogels [51]. Therein, it seemed that the suitable combination of flexible/rigid segments in molecular spacer in cholesteryl gelators is favorable for the gelation of organic solvents. In the present case, the intermolecular steric effect and $\pi-\pi$ stacking of aromatic spacer for in the gel formation process is also obvious, which may increase the ability of the gelator molecules to self-assemble into ordered structures, a necessity for forming organized three-dimensional network structures.

Table 1. Gelation behaviors bolaform cholesteryl compound $\mathrm{CH}-\mathrm{PY}$ at room temperature.

\begin{tabular}{ccc}
\hline Solvents & CH-PY & Label \\
\hline n-Propanol & $\mathrm{S}$ & - \\
Isopropanol & $\mathrm{S}$ & - \\
n-Butanol & $\mathrm{G}(1.5)$ & $\mathrm{a}$ \\
n-Pentanol & $\mathrm{G}(1.5)$ & $\mathrm{b}$ \\
Isopentanol & $\mathrm{G}(2.0)$ & $\mathrm{c}$ \\
Isooctanol & $\mathrm{G}(2.0)$ & $\mathrm{d}$ \\
Acetone & $\mathrm{PS}$ & - \\
Cyclopentanone & $\mathrm{G}(1.5)$ & $\mathrm{e}$ \\
Cyclohexanone & $\mathrm{G}(1.5)$ & $\mathrm{f}$ \\
n-Hexane & $\mathrm{PS}$ & - \\
1,4-Dioxane & $\mathrm{PS}$ & - \\
Benzene & $\mathrm{PS}$ & - \\
Toluene & $\mathrm{PG}$ & - \\
\hline
\end{tabular}


Table 1. Cont.

\begin{tabular}{ccc}
\hline Solvents & CH-PY & Label \\
\hline Nitrobenzene & G $(2.0)$ & $\mathrm{g}$ \\
Aniline & $\mathrm{S}$ & - \\
Ethanolamine & $\mathrm{I}$ & - \\
Ethyl acetate & $\mathrm{I}$ & - \\
n-Butyl acrylate & G $(1.5)$ & $\mathrm{h}$ \\
Acetonitrile & $\mathrm{I}$ & - \\
THF & G $(2.0)$ & $\mathrm{i}$ \\
Pyridine & PS & - \\
Petroleum ether & PS & - \\
DMF & G (1.5) & j \\
\hline
\end{tabular}

DMF: dimethylformamide; THF: tetrahydrofuran; S: solution; PS: partially soluble; G: gel; I: insoluble; for gels, the critical gelation concentrations at room temperature are shown in parentheses, (w/v) $\%$.

\subsection{Morphological Studies of Organogels}

It was well-known that a gelator molecule could construct organized nanostructures, such as nanofibers, nanoribbons, and nanosheets in a supramolecular gel [54-57]. To obtain a visual insight into nanostructures of present gels, the samples of these gels were studied by SEM techniques, as shown in Figure 2. From the present diverse images, it can be easily investigated that the nanostructures of the xerogels in different solvents are significantly different from each other, and the morphologies of the aggregates change from slice, wrinkle, fiber, to rod with change of solvents. Besides, the xerogels of CH-PY in some solvents, such as n-pentanol, cyclopentanone, cyclohexanone, n-butyl acrylate, THF, and DMF, were characterized by AFM, as shown in the supplementary material. From the images, it is interesting to note that morphologies of nanosized fiber, rod, and slice were observed for six xerogels, respectively. At the same time, we have done a comparison test of drying process by freeze-dried method with low temperatures to remove solvents, which can maintain the original structures. The same nanostructures were obtained in both cases. So, the difference of morphologies is attributed to the various assembly modes and forces between gelators and solvents molecules, not the drying process of solvent evaporation. The morphologies of the aggregates shown in the SEM and AFM images may be rationalized by considering a commonly accepted idea that highly directional intermolecular interactions, such as hydrogen bonding or $\pi-\pi$ interactions, favor formation of organized belt or fiber micro/nanostructures [57-60]. The differences of morphologies between different organogels could be mainly due to the different strengths of the hydrophobic force between cholesteryl segments, $\pi-\pi$ stacking and steric hindrance between aromatic spacer, which played an important role in regulating the intermolecular orderly staking and formation of special aggregates. 
Figure 1. Photographs of organogels of CH-PY in different solvents: n-butanol, n-pentanol, isopentanol, isooctanol, cyclopentanone, cyclohexanone, nitrobenzene, n-butyl acrylate, THF, and DMF (from left to right).

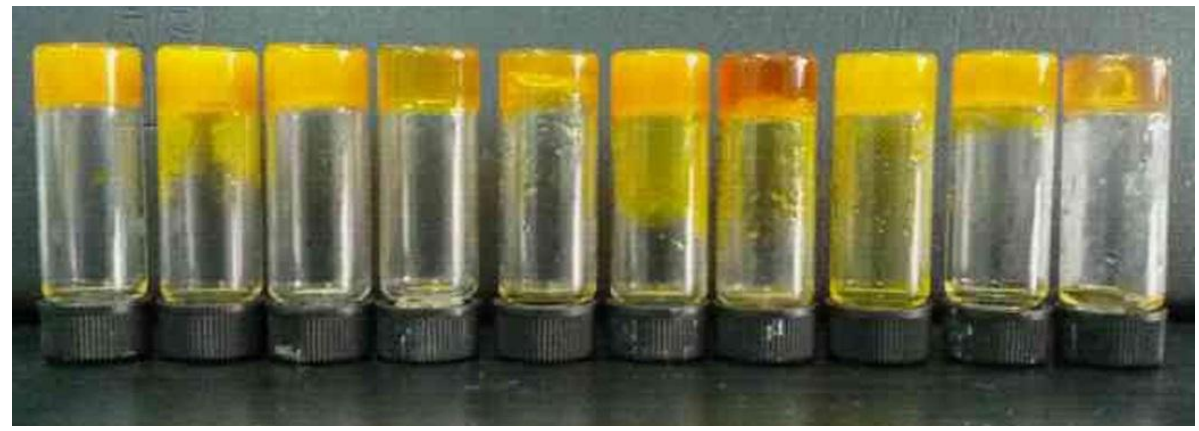

Figure 2. SEM images of xerogels from CH-PY gels: (a-j) n-butanol, n-pentanol, isopentanol, isooctanol, cyclopentanone, cyclohexanone, nitrobenzene, n-butyl acrylate, THF, DMF, respectively.

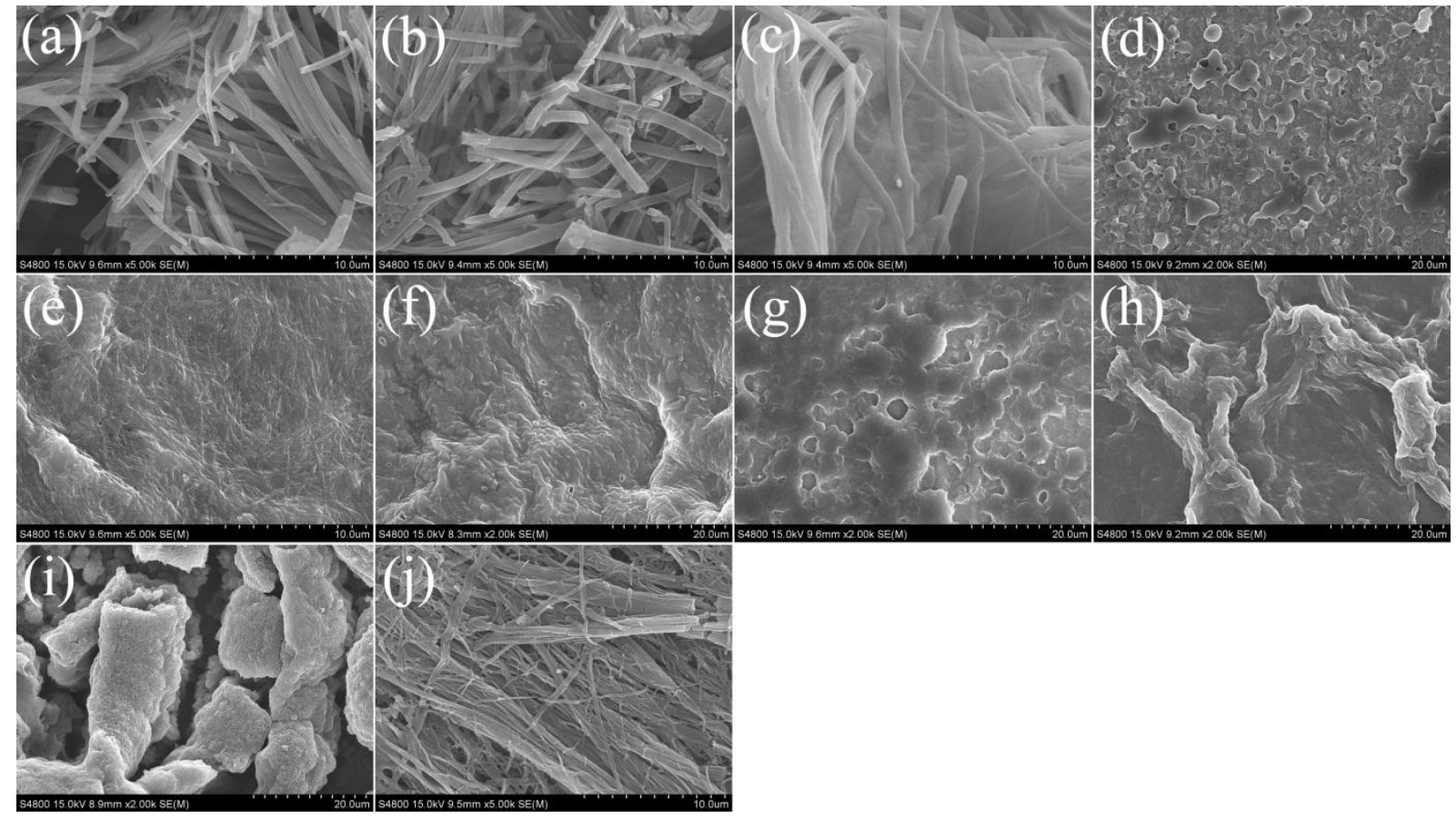

\subsection{Spectral Studies of Organogels}

In addition, with the purpose of investigating the orderly stacking of xerogels nanostructures, XRD of all xerogels from CH-PY gels were measured, as shown in Figure 3. The curve of CH-PY xerogel from n-butyl acrylate showed main peaks in the angle region (20 values, 2.52, 4.40, 6.28, 9.46, and $18.54^{\circ}$ ) corresponding to $d$ values of $3.51,2.01,1.41,0.94$, and $0.48 \mathrm{~nm}$, respectively. As for the curves of $\mathrm{CH}-\mathrm{PY}$ in other solvents, such as n-pentanol and cyclopentanone, the minimum $2 \theta$ values are $3.24^{\circ}$ and $3.72^{\circ}$, corresponding to $d$ values of 2.73 and $2.38 \mathrm{~nm}$, respectively. The change of values can be mainly assigned to the different assembly modes of gelator in various solvents. The XRD results demonstrated again that the solvent had great effects on the assembly modes of the present imide gelator. 
Figure 3. XRD patterns of xerogels from CH-PY gels: (a-j) n-butanol, n-pentanol, isopentanol, isooctanol, cyclopentanone, cyclohexanone, nitrobenzene, n-butyl acrylate, THF, DMF, respectively.

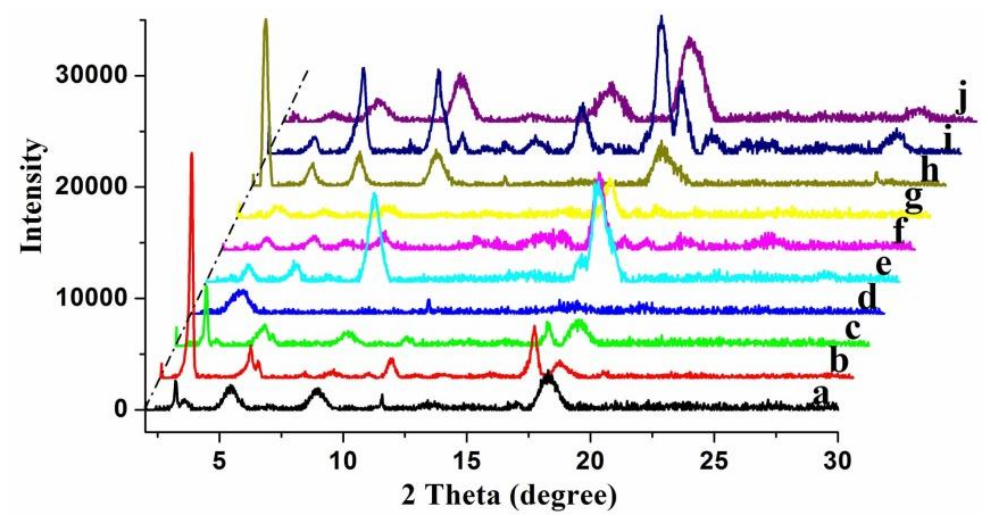

It is well-known that the hydrogen bonding plays an important role in the self-assembly process of organogels $[61,62]$. At present, we have measured the FT-IR spectra of CH-PY in chloroform solution and xerogels in order to further investigate the assembly process, as shown in Figure 4. As for the spectrum of CH-PY in chloroform solution, four main peaks are observed at 3406, 3318, 1723 and $1655 \mathrm{~cm}^{-1}$. These bands can be assigned to the $\mathrm{N}-\mathrm{H}$ stretching, $\mathrm{C}=\mathrm{O}$ stretching of ester, and the amide I band, respectively [52,63]. As far as the xerogels from n-butanol, n-pentanol, isopentanol, n-butyl acrylate, and DMF were concerned, these bands shifted to 3301, 1730 and $1664 \mathrm{~cm}^{-1}$, respectively. As for the xerogels from other solvents, while these bands shifted to 3294 and $1669 \mathrm{~cm}^{-1}$, a new band at $1708 \mathrm{~cm}^{-1}$ appeared in these xerogels. The shift of these bands indicates $\mathrm{H}$-bond formation between intermolecular amide and carbonyl groups in the gel state. Spectra of xerogels in different solvents are different, suggesting the different H-bond and assembly modes of gelator in various solvents. This implied that there were differences in the strength and direction of the intermolecular hydrogen-bond interactions in these xerogels. The present data further verified that the organic solvents can regulate the stacking of the gelator molecules to self-assemble into ordered structures by distinct intermolecular hydrogen bonding.

In addition, the UV-Vis and FL spectra of CH-PY in ethanol solution and xerogels were investigated in order to further character their assembly behaviors, as shown in Figure 5. As for the absorption spectrum of CH-PY in ethanol solution, the absorption peaks appeared at 208, 283, 388, and $447 \mathrm{~nm}$, which could be ascribed to the local $\pi-\pi^{*}$ transition of aromatic ring and charge transfer of conjugated structure in spacer [64,65]. For the spectra of xerogels from different solvents, the latter two absorption bands shifted noticeably to the ranges of 398-402 and 465-482 nm, respectively. The spectral changes suggested that $\mathrm{CH}-\mathrm{PY}$ formed different kinds of J-aggregate-like aggregations in these organized gels. Besides, the FL spectra were also measured to compare this difference. In comparison with the spectrum of ethanol solution with main band at $432 \mathrm{~nm}$, the spectra of xerogels showed main bands in the range of 538-606 nm, indicating different stacking modes of CH-PY in the gels. 
Figure 4. FT-IR spectra of CH-PY in c xerogels $(\mathbf{a}-\mathbf{j})$ : n-butanol, n-pentanol, isopentanol, isooctanol, cyclopentanone, cyclohexanone, nitrobenzene, n-butyl acrylate, THF, DMF, respectively, and in chloroform solution $(\mathrm{k})$.

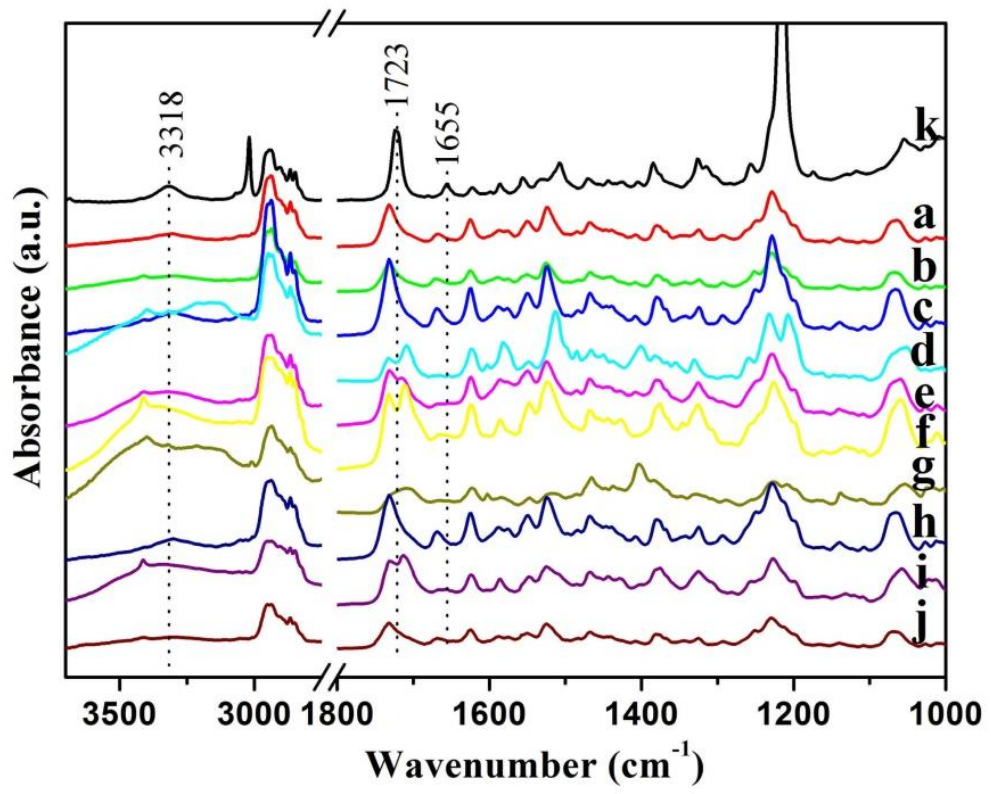

Figure 5. UV-Vis (a) and FL (b) spectra of CH-PY in xerogels (a-j): n-butanol, n-pentanol, isopentanol, isooctanol, cyclopentanone, cyclohexanone, nitrobenzene, n-butyl acrylate, THF, DMF, respectively, and in ethanol solution (k).

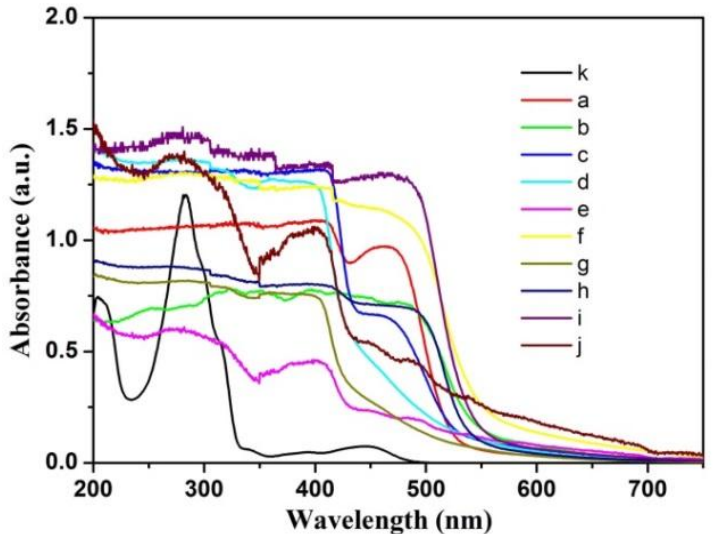

(a)

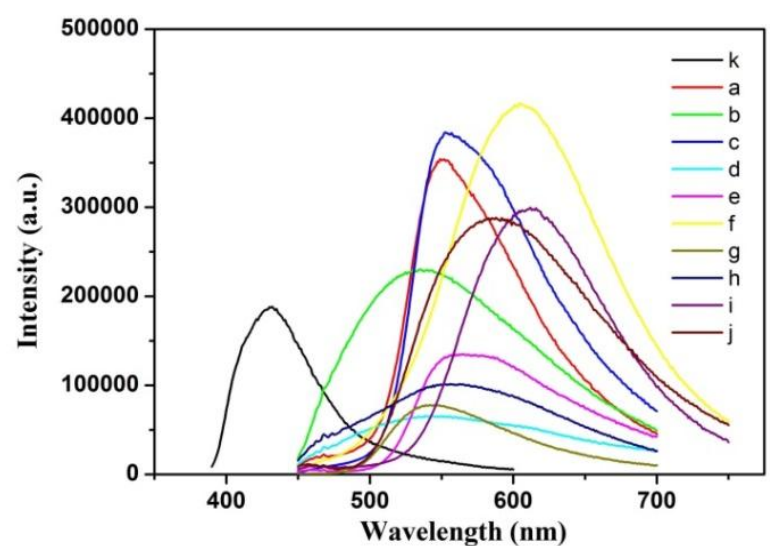

(b)

\subsection{Assembly Modes in Organogels}

Considering the experimental results described above and the hydrogen bonding nature of the organized packing of these organogels as confirmed by FT-IR measurements, some possible assembly modes of the gelator were proposed and schematically shown in Figure 6. As for CH-PY xerogel from n-butyl acrylate, due to the rigidity of conjugated spacer in molecular skeleton and intermolecular forces with solvents, after the intermolecular hydrogen bonding and orderly $\pi-\pi$ stacking, CH-PY molecules have a tendency to self-assemble in mode of stretched stacking. So the repeating unit with length of about $3.5 \mathrm{~nm}$ was obtained, as show in Figure 6a. As for CH-PY xerogels from other 
solvents, such as n-pentanol and cyclopentanone, in comparison with $\pi-\pi$ stacking, the intermolecular forces with solvents seemed more obvious to adjust molecular conformation to self-assemble and form different twisted stacking nanostructures. The obtained experimental values of CH-PY in n-pentanol and cyclopentanone were 2.73 and $2.38 \mathrm{~nm}$, suggesting the twisted conformation in aggregates, shown in Figure 6b. Meanwhile, it should be noted that this phenomenon can be compared with the results of our recent works [50,51]. Therein, functionalized imide derivatives with the different substituent headgroups or spacers, can have a profound effect upon the gelation abilities and the as-formed nanostructures of the studied compounds. Suitable combination of flexible/rigid segments in molecular spacer in cholesteryl gelators is also favorable for the gelation of organic solvents. For the present gelator, the experimental data showed that the conjugated aromatic spacer in the molecular skeleton played a crucial role in the gelation behavior. The strong $\pi-\pi$ stacking and flexible spatial conformation of benzene ring in spacer, as well as in intermolecular forces with solvents, can make the molecules align and stack in an organized way to form various nanostructures. Now, the drug release behaviors generated by the present xerogels in the mixture of Congo red are under investigation to display the relationship between the molecular structures, as-formed nanostructures and properties.

Figure 6. Rational assembly modes of CH-PY organogels in stretched stacking (a) $3.5 \mathrm{~nm}$ and twisted stacking; (b) 2.73 and $2.38 \mathrm{~nm}$, respectively.
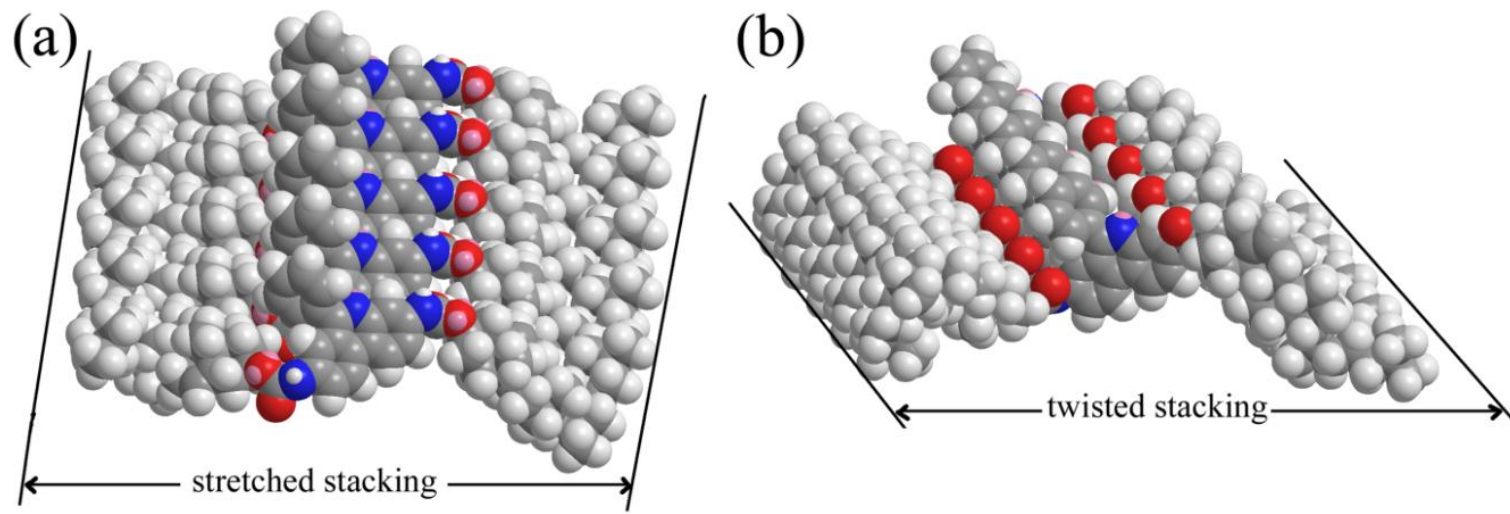

\section{Experimental Section}

\subsection{Materials}

The starting materials, cholesteryl chloroformate and 3,8-diamino-6-phenylphenanthridine were purchased from Alfa Aesar (Tianjin, China) and Chemicals and TCI Chemicals (Shanghai, China), respectively. All other used reagents shown in Table 1, sourced from Beijing Chemicals, were used for analysis purity and were distilled before use. Deionized water was used in all cases. Then, the cholesteryl imide compound was synthesized by similar method according to our previous report [40]. Simply speaking, the synthesis of cholesteryl derivative (abbreviated as CH-PY) was carried out from mixing cholesteryl chloroformate with 3,8-diamino-6-phenylphenanthridine in dried dichloromethane with 2.05:1 molar ratio at the presence of pyridine. After the reaction mixture was stirred for $50 \mathrm{~h}$ at room temperature, the mixture was washed with dilute hydrochloric acid and pure water, respectively. The organic layer was evaporated to dryness. The product was purified by recrystallization in ethanol 
solution as yellow solid. The final product and its abbreviation are shown in Figure 7, which were confirmed by ${ }^{1} \mathrm{H}-\mathrm{NMR}$ and elemental analysis. Ch-PY: ${ }^{1} \mathrm{H}-\mathrm{NMR}\left(400 \mathrm{MHz}, \mathrm{CDCl}_{3}\right): \delta(\mathrm{ppm})$ 0.69-1.97 (82H, m, cholesteryl protons), 2.29-2.51 (4H, d, $\left.\mathrm{CH}_{2}\right), 4.64$ (2H, m, oxycyclohexyl), $5.40(2 \mathrm{H}, \mathrm{s}$, alkenyl), $6.76(2 \mathrm{H}, \mathrm{s}, \mathrm{CONH}), 7.64(2 \mathrm{H}, \mathrm{s}$, benzene ring), $7.83(2 \mathrm{H}, \mathrm{s}$, benzene ring), $8.10\left(2 \mathrm{H}\right.$, s, benzene ring), 8.52-8.84 (5H, m, benzene ring). Anal. Calcd. for $\mathrm{C}_{75} \mathrm{H}_{103} \mathrm{~N}_{3} \mathrm{O}_{4}: \mathrm{C}, 81.11$; H, 9.35; N, 3.78. Found: C, 81.45; H, 9.42; N, 3.79 .

Figure 7. Structure and abbreviation of bolaform cholesteryl imide derivative with aromatic spacer.

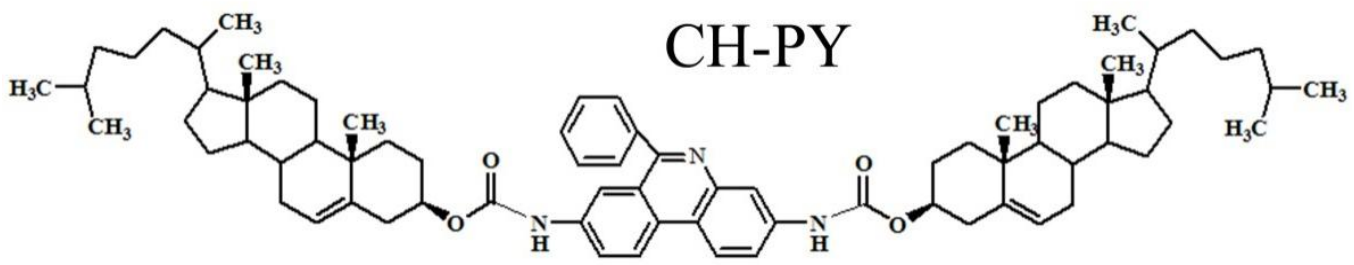

\subsection{Gel Preparation}

The present cholesteryl imide derivative with aromatic spacer was tested to prepare possible organogels according to a simple procedure. Firstly, a weighted amount of imide compound and a measured volume of selected pure organic solvent were placed into a sealed glass bottle and the solution ultrasonicated in a sonic bath for $15 \mathrm{~min}$ in order to obtain good dispersion. After that, the solution heated in a water bath at temperatures of $80{ }^{\circ} \mathrm{C}$ for $15 \mathrm{~min}$. Then, the solution was cooled to room temperature in air and the test bottle was inversed to see if a gel was formed. At this stage, G, S, PS, and I were denoted to character the states of imide derivative, indicating gel, solution, a few precipitate and insoluble systems, respectively. Critical gelation concentration refers to the minimum concentration of the gelator for gel formation.

\subsection{Characterization}

These prepared organogels under the critical gelation concentration were dried by a vacuum pump for more than $12 \mathrm{~h}$ to remove solvents and form xerogels. Then, the obtained xerogel samples were attached to different substrates, such as mica, copper foil, glass, and $\mathrm{CaF}_{2}$ slice for morphological and spectral investigation, respectively. AFM data were measured by using Nanoscope VIII Multimode Scanning Probe Microscope (Veeco Instrument, Plainview, NY, USA) with silicon cantilever probes. All AFM images were shown in the height mode without any image processing except flattening. SEM images of the xerogels were measured on a Hitachi S-4800 field emission scanning electron microscopy with the accelerating voltage of 5-15 kV. For SEM measurement, the samples were coated on copper foil fixed by conductive adhesive tape and shielded by gold nanoparticles. The XRD was measured by using a Rigaku D/max 2550PC diffractometer (Rigaku Inc., Tokyo, Japan) with $\mathrm{CuK} \alpha$ radiation wavelength of $0.1542 \mathrm{~nm}$ under a voltage of $40 \mathrm{kV}$ and a current of $200 \mathrm{~mA}$. UV-vis spectra were obtained by a Jasco UV-530 spectrophotometer (Jasco Corp., Tokyo, Japan). Fluorescence spectra were acquired on a FLS 920 spectrofluorometer (Edinburgh Instruments, Edinburgh, UK) using $1 \mathrm{~cm}$ quartz cuvettes or quartz plates. All fluorescence spectra were corrected for the sensitivity 
of the photo-multiplier tube. FT-IR spectra were obtained by Nicolet is/10 FT-IR spectrophotometer from Thermo Fisher Scientific Inc. by an average 32 scans and at a resolution of $4 \mathrm{~cm}^{-1}$.

\section{Conclusions}

In summary, a bolaform cholesteryl imide compound with conjugated aromatic spacer has been designed and synthesized. The gelation behaviors in 23 kinds of organic solvents have been investigated. The morphologies and assembly modes of formed organogels can be regulated by changing organic solvents. Morphological studies revealed that the gelator molecules self-assemble into different aggregates, from wrinkle and belt to fiber with the change of spacers and solvents. Spectral studies indicated that there existed different H-bond formations between imide groups and assembly modes, depending on the solvents. The prepared nanostructures have potential applications in nanomaterial fields. These results afford useful information for the design and development of new versatile low molecular mass organogelators and soft matter.

\section{Acknowledgments}

This work was financially supported by the National Natural Science Foundation of China (grant No. 21207112), the Natural Science Foundation of Hebei Province (grant Nos. B2012203060, B2013203108), the China Postdoctoral Science Foundation (grant Nos. 2011M500540, 2012M510770, 2013T60265), and the Science Foundation for the Excellent Youth Scholars from Universities and Colleges of Hebei Province (grant Nos. Y2011113, YQ2013026). Helpful review and discussions with Fang Zhang is highly acknowledged.

\section{Conflicts of Interest}

The authors declare no conflict of interest.

\section{References}

1. Matsumoto, T.; Mizuno, A.; Kashiwagi, M.; Yoshida, S.; Sasaki, J.; Nakano, T. Cell-based fabrication of organic/inorganic composite gel material. Materials 2011, 4, 327-338.

2. Itoh, N. Electrochemical light-emitting gel. Materials 2010, 3, 3729-3739.

3. Suzuki, M.; Hanabusaa, K. L-lysine-based low-molecular-weight gelators. Chem. Soc. Rev. 2009, 38, 967-975.

4. Cravotto, G.; Cintas, P. Molecular self-assembly and patterning induced by sound waves. The case of gelation. Chem. Soc. Rev. 2009, 38, 2684-2697.

5. Piepenbrock, M.O.; Lloyd, G.O.; Clarke, N.; Steed, J.W. Metal- and anion-binding supramolecular gels. Chem. Rev. 2010, 110, 1960-2004.

6. George, M.; Weiss, R.G. Molecular organogels. Soft matter comprised of low-molecular-mass organic gelators and organic liquids. Acc. Chem. Res. 2006, 39, 489-497.

7. Ohsedo, Y.; Miyamoto, M.; Tanaka, A.; Watanabe, H. Synthesis of a new squarylium alkylamide family and its organogelation ability. New J. Chem. 2013, 37, 2874-2880. 
8. Boiani, M.; Baschieri, A.; Cesari, C.; Mazzoni, R.; Stagni, S.; Zacchini, S.; Sambri, L. A new tetraarylcyclopentadienone based low molecular weight gelator: Synthesis, self-assembly properties and anion recognition. New J. Chem. 2012, 36, 1469-1478.

9. Sambri, L.; Cucinotta, F.; De Paoli, G.; Stagnic, S.; De Colab, L. Ultrasound-promoted hydrogelation of terpyridine derivatives. New J. Chem. 2010, 34, 2093-2096.

10. Foster, J.A.; Piepenbrock, M.O.M.; Lloyd, G.O.; Clarke, N.; Howard, J.A.K.; Steed, J.W. Anion-switchable supramolecular gels for controlling pharmaceutical crystal growth. Nat. Chem. 2010, 2, 1037-1043.

11. Lloyd, G.O.; Piepenbrock, M.O.M.; Foster, J.A.; Clarke, N.; Steed, J.W. Anion tuning of chiral bis(urea) low molecular weight gels. Soft Matter 2012, 8, 204-216.

12. Wu, S.; Gao, J.; Emge, T.J.; Rogers, M.A. Influence of solvent on the supramolecular architectures in molecular gels. Soft Matter 2013, 9, 5942-5950.

13. Jiang, Y.; Zeng, F.; Gong, R.; Guo, Z.; Chen, C.; Wan, X. A multi-stimuli responsive organogel based on a tetrapeptide-dithienylcyclopentene conjugate. Soft Matter 2013, 9, 7538-7544.

14. Delbecq, F.; Tsujimoto, K.; Ogue, Y.; Endo, H.; Kawai, T. N-stearoyl amino acid derivatives: Potent biomimetic hydro/organogelators as templates for preparation of gold nanoparticles. J. Colloid Interface Sci. 2013, 390, 17-24.

15. Svobodová, H.; Noponen, V.; Kolehmainen, E.; Sievänen, E. Recent advances in steroidal supramolecular gels. RSC Adv. 2012, 2, 4985-5007.

16. Li, J.; Kuang, Y.; Gao, Y.; Du, X.; Shi, J.; Xu, B. D-Amino acids boost the selectivity and confer supramolecular hydrogels of a nonsteroidal anti-inflammatory drug (NSAID). J. Am. Chem. Soc. 2013, 135, 542-545.

17. Liu, K.; Liu, T.; Chen, X.; Sun, X.; Fang, Y. Fluorescent films based on molecular-gel networks and their sensing performances. ACS Appl. Mater. Interfaces 2013, 5, 9830-9836.

18. Basrur, V.R.; Guo, J.; Wang, C.; Raghavan, S.R. Synergistic gelation of silica nanoparticles and a sorbitol-based molecular gelator to yield highly-conductive free-standing gel electrolytes. ACS Appl. Mater. Interfaces 2013, 5, 262-267.

19. Chen, X.; Liu, K.; He, P.; Zhang, H.; Fang, Y. Preparation of novel W/O gel-emulsions and their application in the preparation of low-density materials. Langmuir 2012, 28, 9275-9281.

20. Su, Y.S.; Liu, J.W.; Jiang, Y.; Chen, C.F. Assembly of a self-complementary monomer: Formation of supramolecular polymer networks and responsive gels. Chem. Eur. J. 2011, 17, 2435-2441.

21. Liu, J.W.; Yang, Y.; Chen, C.F.; Ma, J.T. Novel anion-tuning supramolecular gels with dual-channel response: Reversible sol-gel transition and color changes. Langmuir 2010, 26, 9040-9044.

22. Xing, P.; Sun, T.; Li, S.; Hao, A.; Su, J.; Hou, Y. An instant-formative heat-set organogel induced by small organic molecules at a high temperature. Colloid Surf. A Physicochem. Eng. Asp. 2013, $421,44-50$.

23. Kuroiwa, K.; Shibata, T.; Takada, A.; Nemoto, N.; Kimizuka, N. Heat-set gel-like networks of lipophilic $\mathrm{Co}(\mathrm{II})$ triazole complexes in organic media and their thermochromic structural transitions. J. Am. Chem. Soc. 2004, 126, 2016-2021. 
24. Aldred, M.P.; Eastwood, A.J.; Kelly, S.M.; Vlachos, P.; Contoret, A.E.A.; Farrar, S.R.; Mansoor, B.; O’Neill, M.; Tsoi, W.C. Light-emitting fluorene photoreactive liquid crystals for organic electroluminescence. Chem. Mater. 2004, 16, 4928-4936.

25. Yan, N.; Xu, Z.; Diehn, K.K.; Raghavan, S.R.; Fang, Y.; Weiss, R.G. Pyrenyl-linker-glucono gelators. correlations of gel properties with gelator structures and characterization of solvent effects. Langmuir 2013, 29, 793-805.

26. George, S.J.; Ajayaghosh, A. Self-assembled nanotapes of oligo(p-phenylene vinylene)s: Sol-gel-controlled optical properties in fluorescent $\pi$-electronic gels. Chem. Eur. J. 2005, 11, 3217-3227.

27. Ajayaghosh, A.; Chithra, P.; Varghese, R. Self-assembly of tripodal squaraines: Cation-assisted expression of molecular chirality and change from spherical to helical morphology. Angew. Chem. Int. Ed. 2007, 46, 230-233.

28. Dai, H.; Chen, Q.; Qin, H.; Guan, Y.; Shen, D.; Hua, Y.; Tang, Y.; Xu, J. A temperature-responsive copolymer hydrogel in controlled drug delivery. Macromolecules 2006, 39, 6584-6589.

29. Zhang, X.; Chen, Z.; Wurthner, F. Morphology control of fluorescent nanoaggregates by co-self-assembly of wedge- and dumbbell-shaped amphiphilic perylene bisimides. J. Am. Chem. Soc. 2007, 129, 4886-4887.

30. Boerakker, M.J.; Botterhuis, N.E.; Bomans, P.H.H.; Frederik, P.M.; Meijer, E.M.; Nolte, R.J.M.; Sommerdijk, N.A.J.M. Aggregation behavior of giant amphiphiles prepared by cofactor reconstitution. Chem. Eur. J. 2006, 12, 6071-6080.

31. Iwanaga, K.; Sumizawa, T.; Miyazaki, M.; Kakemi, M. Characterization of organogel as a novel oral controlled release formulation for lipophilic compounds. Int. J. Pharm. 2010, 388, 123-128.

32. Lofman, M.; Koivukorpi, J.; Noponen, V.; Salo, H.; Sievanen, E. Bile acid alkylamide derivatives as low molecular weight organogelators: Systematic gelation studies and qualitative structural analysis of the systems. J. Colloid Interface Sci. 2011, 360, 633-644.

33. Xin, F.; Zhang, H.; Hao, B.; Sun, T.; Kong, L.; Li, Y.; Hou, Y.; Li, S.; Zhang, Y.; Hao, A. Controllable transformation from sensitive and reversible heat-set organogel to stable gel induced by sodium acetate. Colloid Surf. A Physicochem. Eng. Asp. 2012, 410, 18-22.

34. Tao, Z.G.; Zhao, X.; Jiang, X.K.; Li, Z.T. A hexaazatriphenylene-based organogel that responds to silver(I) with high selectivity under aqueous condition. Tetrahedron Lett. 2012, 53, 1840-1842.

35. Miyamoto, K.; Jintoku, H.; Sawada, T.; Takafuji, M.; Sagawa, T.; Ihara, H. Informative secondary chiroptics in binary molecular organogel systems for donor-acceptor energy transfer. Tetrahedron Lett. 2011, 52, 4030-4035.

36. Bastiat, G.; Plourde, F.; Motulsky, A.; Furtos, A.; Dumont, Y.; Quirion, R.; Fuhrmann, G.; Leroux, J.C. Tyrosine-based rivastigmine-loaded organogels in the treatment of Alzheimer's disease. Biomaterials 2010, 31, 6031-6038.

37. Jiao, T.F.; Wang, R.X.; Zhang, Q.R.; Yan, X.B.; Zhou, J.X.; Gao, F.M. Nanostructures and substituent alkyl chains effect on assembly of organogels based on some glutamic acid diethyl ester imide derivatives. Curr. Nanosci. 2013, 9, 536-542.

38. Bauer, M.; Charitat, T.; Fajolles, C.; Fragneto, G.; Daillant, J. Insertion properties of cholesteryl cyclodextrins in phospholipid membranes: A molecular study. Soft Matter 2012, 8, 942-953. 
39. Castruita, G.; Garcia, V.; Arias, E.; Moggio, I.; Ziolo, R.; Ponce, A.; Gonzalez, V.; Haley, J.E.; Flikkema, J.L.; Cooper, T. Synthesis and microwave electromagnetic properties of nanosized titanium nitride. J. Mater. Chem. 2012, 22, 3370-3376.

40. Yu, X.; Cao, X.; Chen, L.; Lan, H.; Liu, B.; Yi, T. Thixotropic and self-healing triggered reversible rheology switching in a peptide-based organogel with a cross-linked nano-ring pattern. Soft Matter 2012, 8, 3329-3334.

41. Jung, J.H.; Kobayashi, H.; Masuda, M.; Shimizu, T.; Shinkai, S. Helical ribbon aggregate composed of a crown-appended cholesterol derivative which acts as an amphiphilic gelator of organic solvents and as a template for chiral silica transcription. J. Am. Chem. Soc. 2001, 123, 8785-8789.

42. Jung, J.H.; Kobayashi, H.; van Bommel, K.J.C.; Shinkai, S.; Shimizu, T. Creation of novel helical ribbon and double-layered nanotube $\mathrm{TiO} 2$ structures using an organogel template. Chem. Mater. 2002, 14, 1445-1447.

43. Sugiyasu, K.; Fujita, N.; Shinkai, S. Fluorescent organogels as templates for sol-gel transcription toward creation of optical nanofibers. J. Mater. Chem. 2005, 15, 2747-2754.

44. Jong, J.H.; Nakashima, K.; Shinkai, S. Preparation of ultrastable mesoporous silica using a phenanthroline-appended cholesterol organogelator as a template. Nano Lett. 2001, 1, 145-148.

45. Jong, J.H.; Ono, Y.; Shinkai, S. Novel silica structures which are prepared by transcription of various superstructures formed in organogels. Langmuir 2000, 16, 1643-1649.

46. Hou, X.Y.; Gao, D.; Yan, J.L.; Ma, Y.; Liu, K.Q.; Fang, Y. Novel dimeric cholesteryl derivatives and their smart thixotropic gels. Langmuir 2011, 27, 12156-12163.

47. He, P.L.; Liu, J.; Liu, K.Q.; Ding, L.P.; Yan, J.L.; Gao, D.; Fang, Y. Preparation of novel organometallic derivatives of cholesterol and their gel-formation properties. Colloid Surf. A Physicochem. Eng. Asp. 2010, 362, 127-134.

48. Peng, J.X.; Liu, K.Q.; Liu, J.; Zhang, Q.H.; Feng, X.L.; Fang, Y. New dicholesteryl-based gelators: Chirality and spacer length effect. Langmuir 2008, 24, 2992-3000.

49. Peng, J.X.; Liu, K.Q.; Liu, X.F.; Xia, H.Y.; Liu, J.; Fang, Y. New dicholesteryl-based gelators: Gelling ability and selective gelation of organic solvents from their mixtures with water at room temperature. New J. Chem. 2008, 32, 2218-2224.

50. Jiao, T.F.; Wang, Y.J.; Gao, F.Q.; Zhou, J.X.; Gao, F.M. Photoresponsive organogel and organized nanostructures of cholesterol imide derivatives with azobenzene substituent groups. Prog. Nat. Sci. 2012, 22, 64-70.

51. Jiao, T.F.; Gao, F.Q.; Zhang, Q.R.; Zhou, J.X.; Gao, F.M. Spacer effect on nanostructures and self-assembly in organogels via some bolaform cholesteryl imide derivatives with different spacers. Nanoscale Res. Lett. 2013, 8, 406.

52. Jiao, T.F.; Gao, F.Q.; Wang, Y.J.; Zhou, J.X.; Gao, F.M.; Luo, X.Z. Supramolecular gel and nanostructures of bolaform and trigonal cholesteryl derivatives with different aromatic spacers. Curr. Nanosci. 2012, 8, 111-116.

53. Zhang, J.; Lakowicz, J.R. Enhanced luminescence of phenyl-phenanthridine dye on aggregated small silver nanoparticles. J. Phys. Chem. B 2005, 109, 8701-8706. 
54. Duan, P.; Qin, L.; Zhu, X.; Liu, M. Hierarchical self-assembly of amphiphilic peptide dendrons: Evolution of diverse chiral nanostructures through hydrogel formation over a wide $\mathrm{pH}$ range. Chem. Eur. J. 2011, 17, 6389-6395.

55. Zhu, G.Y.; Dordick, J.S. Solvent effect on organogel formation by low molecular weight molecules. Chem. Mater. 2006, 18, 5988-5995.

56. Wu, J.C.; Yi, T.; Zou, Y.; Xia, Q.; Shu, T.; Liu, F.; Yang, Y.H.; Li, F.Y.; Chen, Z.G.; Zhou, Z.G.; et al. Gelation induced reversible syneresis via structural evolution. J. Mater. Chem. 2009, 19, 3971-3978.

57. Zhu, X.; Duan, P.; Zhang, L.; Liu, M. Regulation of the chiral twist and supramolecular chirality in co-assemblies of amphiphilic L-glutamic acid with bipyridines. Chem. Eur. J. 2011, 17, 3429-3437.

58. Yang, H.; Yi, T.; Zhou, Z.; Zhou, Y.; Wu, J.; Xu, M.; Li, F.; Huang, C. Switchable fluorescent organogels and mesomorphic superstructure based on naphthalene derivatives. Langmuir 2007, $23,8224-8230$.

59. Nayak, M.K. Functional organogel based on a hydroxyl naphthanilide derivative and aggregation induced enhanced fluorescence emission. J. Photochem. Photobiol. A Chem. 2011, 217, 40-48.

60. Shimizu, T.; Masuda, M. Stereochemical effect of even-odd connecting links on supramolecular assemblies made of 1-glucosamide bolaamphiphiles. J. Am. Chem. Soc. 1997, 119, 2812-2818.

61. Li, Y.G.; Wang, T.Y.; Liu, M.H. Ultrasound induced formation of organogel from a glutamic dendron. Tetrahedron 2007, 63, 7468-7473.

62. Kogiso, M.; Ohnishi, S.; Yase, K.; Masuda, M.; Shimizu, T. Dicarboxylic oligopeptide bola-amphiphiles: Proton-triggered self-assembly of microtubes with loose solid surfaces. Langmuir 1998, 14, 4978-4986.

63. Wang, T.Y.; Li, Y.G.; Liu, M.H. Gelation and self-assembly of glutamate bolaamphiphiles with hybrid linkers: Effect of the aromatic ring and alkyl linkers. Soft Matter 2009, 5, 1066-1073.

64. Jiao, T.F.; Liu, M.H. Substitution controlled molecular orientation and nanostructure in the Langmuir-Blodgett films of a series of amphiphilic naphthylidene-containing Schiff base derivatives. J. Colloid Interface Sci. 2006, 299, 815-822.

65. Chichak, K.; Jacquemard, U.; Branda, N.R. The construction of (salophen)ruthenium(II) assemblies using axial coordination. Eur. J. Inorg. Chem. 2002, 2, 357-368.

(C) 2013 by the authors; licensee MDPI, Basel, Switzerland. This article is an open access article distributed under the terms and conditions of the Creative Commons Attribution license (http://creativecommons.org/licenses/by/3.0/). 